\title{
ANC experiments for nuclear astrophysics
}

\author{
G. D’Agata ${ }^{1}$, V. Burjan ${ }^{1}$, J. Mrázek ${ }^{1}$, V. Glagolev' ${ }^{1}$, A. I. Kilic ${ }^{1}$, V. Kroha ${ }^{1}$, M. La Cognata ${ }^{2}$, L. \\ Lamia $^{2,3}$, S. Palmerini ${ }^{4,5}$, Š. Piskoř ${ }^{1}$, R. G. Pizzone ${ }^{2}$, G. G. Rapisarda ${ }^{2}$, S. Romano ${ }^{2,3}$, I. Siváček ${ }^{1}$, \\ C. Spitaleri ${ }^{2}, R$. Spartá ${ }^{2}$, and $A$. Tumino ${ }^{2,6}$ \\ ${ }^{1}$ Nuclear Physics Institute of the Czech Academy of Sciences, 25068 Řež, Czech Republic \\ 2 INFN - Laboratori Nazionali del Sud, Via Santa Sofia 62, 95123, Catania, Italy \\ ${ }^{3}$ Dipartimento di Fisica e Astronomia, Università degli Studi di Catania, Via S. Sofia, 64, 95123, Catania, \\ Italy \\ ${ }^{4}$ Dipartimento di Fisica e Geologia, Università degli Studi di Perugia, via A. Pascoli s/n, 06123, Perugia, Italy \\ 5 INFN-sezione di Perugia, via A. Pascoli s/n, 06123, Perugia, Italy \\ ${ }^{6}$ Facoltà di Ingegneria ed Architettura, Kore University, Viale delle Olimpiadi, 1, I-94100 Enna, Italy
}

\begin{abstract}
Among the indirect methods to determine nuclear cross-section present in literature, the so-called Asymptotic Normalization Coefficient (ANC) has proven to be useful in retrieving the direct part of a radiative capture cross-section in reactions of interest for astrophysics. In this work, the method will be presented, and some results obtained in collaboration between NPI CAS and INFN-LNS will be presented.
\end{abstract}

\section{Introduction}

Experimental measurements of reactions of relevance for astrophysics are a big challenge for experimentalists due to the presence of the Coulomb barrier between charged particles: in fact, it highly decreases the cross-section in the energy range of interest, that usually is between few keV and some MeV. It is therefore necessary, when direct methods are not feasible, to use indirect ones. Among these, the Asymptotic Normalization Coefficient (ANC) method allows to retrieve the ANC of the overlap function for the bound-state wave functions in the initial and final states $[1,2]$. Such a method is widely used under the condition that the reaction of interest is peripheral. This is, for example, the case of radiative captures involving $\mathrm{p}, \mathrm{n}$ and $\alpha$-particles in stellar environment. In the following work the method will be presented, marking the key points of the theoretical framework and the advantages of its usage. Then an experimental overview of the method will be given, using as a benchmark the ongoing data analysis for the ${ }^{26} \mathrm{Mg}(\mathrm{d}, \mathrm{p}){ }^{27} \mathrm{Mg}$ reaction: this reaction is interesting due to its mirror, ${ }^{26} \mathrm{Si}(\mathrm{p}, \gamma){ }^{27} \mathrm{P}$, considered to be important as a constraint to the production of ${ }^{26} \mathrm{Al}$ and ${ }^{26 m} \mathrm{Al}$. The production of both is among the most debated issues concerning novae and $\mathrm{x}$-ray bursts[3, 4].

\section{The ANC method}

The ANC method, as stated in the introduction, is usefull to study direct capture reactions of astrophysical interest by means of the study of a direct transfer reaction. It allows to study reactions 
involving protons [5], neutrons [6] or $\alpha$-particles [7] and it has also proven to be useful to investigate subthreshold resonances [8-10].

In the general case, this kind of reactions can be considered (as sketched figure 1) as $X+A \rightarrow Y+B$, where $X=Y+a, B=A+a$, with $a$ is the transferred particle.

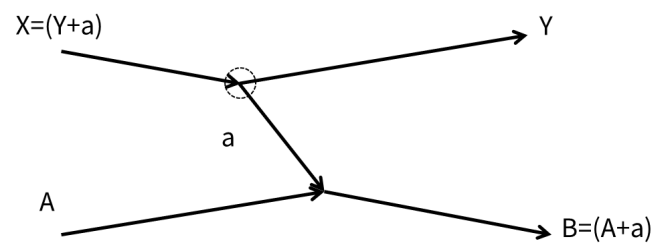

Figure 1. Sketch of a typical transfer reaction.

For that case, the Distorted Wave Born Approximation (DWBA) leads to an approximation for the transition amplitude between the states before and after the reaction. Here, the matrix element for the transition can be written as

$$
M=\left\langle\chi_{f}^{(-)} I_{A a}^{B}|\Delta V| I_{Y a}^{X} \chi_{i}^{(+)}\right\rangle
$$

where $I_{A a}^{B}$ and $I_{Y a}^{X}$ are the radial overlap integral of the two systems $Y+a$ and $A+a$ with the nuclei $B$ and $X$ respectively, $\chi_{i}^{(+)}$and $\chi_{f}^{(-)}$are the distorted waves for the entrance and exit channels, and $\Delta V$ is the perturbative part of the total potential, composed by a real $(V)$ and an optical $(U)$ part $(\Delta V=V-U)$. In general, for the nuclear process between two particles $a+b \rightarrow c$, with $c=(a b)$, both the overlap functions in equation 1 can be written using a quantity known as spectroscopic factor (SF) - in general $\mathrm{S}_{a b}$ - that has been widely used in the last fifty years to estimate the occupation of the orbitals. The overlap funcion of the bound state wave functions of particles $a, b$ and $c, \mathrm{I}_{a b l_{c} j_{c}}^{c}$, can be written as:

$$
I_{a b, l_{c}, j_{c}}^{c}\left(r_{a b}\right)=S_{a b, l_{c}, j_{c}}^{1 / 2} \phi_{n_{c}, l_{c}, j_{c}}\left(r_{a b}\right)
$$

with $\phi_{n_{c} l_{c} j_{c}}\left(r_{a b}\right)$ as the bound state wave function [11].

Regarding the SF, the description of the cross-section depends on the binding portential and optical model parameters (OMP)[11]. The binding potential is adjusted according to the binding energy to reproduce the bound state, while the OMP are usually extracted from elastic scattering. In general then the SF is sensitive to the selection of the optical model adopted, and different families of those can reproduce the angular distributions retrieved from experimental data [12].

By means of DWBA calculation it is in the end possible to reproduce the experimental angular distribution:

$$
\frac{d \sigma^{\exp }}{d \Omega}=\sum_{j_{B}, j_{X}} S_{A a, l_{B}, j_{B}} S_{Y a, l_{X} j_{X}} \sigma_{l_{B}, j_{b}, l_{X}, j_{X}}^{D W}
$$

where $\frac{d \sigma}{d \Omega}$ and $\sigma_{l_{B} j_{b} l_{X} j_{X}}^{D W}$ are related by the two SF for the entrance and exit channel: one of those is usually known, while the other is left to be determined.

In 1990 it was pointed out by Mukhamedzhanov and Timofeyuk[13] that the direct part of the radiative cross-section at low energies usually contains the same radial overlap integral ( $I_{a b}^{c}$ in general) as a direct transfer reaction, and that happens because such capture occurs usually at large distances from the nucleus, where the behaviour can be called Asymptotic. The asymptotic behaviour can be used to 
determine the cross-section of reactions of interest in astrophysics, and in this case is possible to write both the radial overlap functions (Eq. 1) and bound-state wave functions (Eq. 2) as it follows:

$$
\begin{gathered}
I_{A a, l_{c}, j_{c}}^{B}\left(r_{a b}\right) \stackrel{r_{a b}>R_{n}}{\longrightarrow} C_{a b, l_{b}, j_{b}}^{c} \frac{W_{-\eta, l_{c}+\frac{1}{2}}\left(2 k_{a b} r_{a b}\right)}{r_{a b}} \\
\phi_{a b, l_{c}, j_{c}}\left(r_{a b}\right) \stackrel{r_{a b}>R_{n}}{\longrightarrow} b_{a b, l_{c}, j_{c}}^{c} \frac{W_{-\eta, l_{c}+\frac{1}{2}}\left(2 k_{a b} r_{a b}\right)}{r_{a b}}
\end{gathered}
$$

In both equations (4) and (5) $W_{-\eta, l_{c}+\frac{1}{2}}$ stands for the Whittaker function, well known to have its asymptotic behaviour as $W_{-\eta, l_{c}+\frac{1}{2}}\left(-2 k_{I} r\right) \rightarrow e^{-k_{l} r+\eta_{I} \ln \left(2 k_{I}\right)}$. In equation 4 and 5 for the first time the coefficient $b$ and $C$ appear: those are the so-called single-particle ANC (SPANC) and ANC coefficients, respectively. Finally, by substitution of equations (4) and (5) in (3), we obtain:

$$
\frac{d \sigma}{d \Omega}=\sum_{j_{B}, j_{X}}\left(C_{A a, l_{B}, j_{B}}^{B}\right)^{2}\left(C_{Y a, l_{X}, j_{X}}^{X}\right)^{2} \frac{\sigma_{l_{B}, j_{B}, l_{X}, j_{X}}^{D W B{ }_{1}}}{b_{A a, l_{B}, j_{B}}^{2} b_{Y a, l_{X}, j_{X}}^{2}}=\sum_{j_{B}, j_{X}}\left(C_{A a, l_{B}, j_{B}}^{B}\right)^{2}\left(C_{Y a, l_{X}, j_{X}}^{X}\right)^{2} R_{l_{B}, j_{B}, l_{X}, j_{X}}
$$

In the end, using DWBA, we are able to retrieve the ANC's for the direct part of the cross-section for reactions of astrophysical interest. This approach is useful because the ANC's have a small dependence on the chosen nucleon binding potential (see Fig.11 in [11], for example), so the ratio $R_{l_{B}, j_{B}, l_{X}, j_{X}}$ in equation 6 has also small dependence on $b^{2}$.

\section{An experimental example: the ${ }^{26} \mathrm{Si}(\mathrm{p}, \gamma)^{27} \mathrm{P}$ reaction}

One of the latest experiments meant to apply the ANC method at NPI CAS has been the study of the ${ }^{26} \mathrm{Si}(\mathrm{p}, \gamma)^{27} \mathrm{P}$ reaction, that is supposed to be of some importance in the framework of the production of ${ }^{26} \mathrm{Al}$. This reaction is studied using the mirror pair of ${ }^{26} \mathrm{Si},{ }^{26} \mathrm{Mg}$ : in particular, the reaction ${ }^{26} \mathrm{Mg}(\mathrm{d}, \mathrm{p}){ }^{27} \mathrm{Mg}$ is used with the aim to extract ANC's for the ${ }^{26} \mathrm{Mg}(\mathrm{n}, \gamma){ }^{27} \mathrm{Mg}$, and then using the mirror pair properties $[14,15]$ to obtain information on the ANC of the reaction of interest.

\subsection{Astrophysical motivation}

In the last years, the production of ${ }^{26} \mathrm{Al}$ has been matter of debate for scientists: it is, in fact clearly observable in novae and $\mathrm{x}$-ray bursts $[3,4]$. Its production chain seems to be dominated by the ${ }^{24} \mathrm{Mg}(\mathrm{p}, \gamma)^{25} \mathrm{Al}\left(\beta^{+}\right)^{25} \mathrm{Mg}(\mathrm{p}, \gamma)^{26} \mathrm{Al}$, but the scenario has been strongly complicated by the presence of the widely known, short-lived isomer of ${ }^{26} \mathrm{Al}\left(\mathrm{T}_{1 / 2}=6.34 \mathrm{~s}\right)$. The ratio between ground and isomeric states should be known to better understand the observed wide and intense distribution of ${ }^{26} \mathrm{Al}$ through the galactic plane [16].

A possible bypass of the chain mentioned above is represented by the ${ }^{25} \mathrm{Al}(\mathrm{p}, \gamma)^{26} \mathrm{Si}(\mathrm{p}, \gamma){ }^{27} \mathrm{P}$ one, and so the ${ }^{27} \mathrm{P}$ production has been considered of some importance as a possible competitive channel of the ${ }^{26} \mathrm{Al}$ production. Furthermore, the isomeric state ${ }^{26 m} \mathrm{Al}$ can be produced via the ${ }^{25} \mathrm{Al}(\mathrm{p}, \gamma){ }^{26} \mathrm{Si}\left(\beta^{+}\right){ }^{26 m} \mathrm{Al}$. In this scenario the reaction ${ }^{26} \mathrm{Si}(\mathrm{p}, \gamma){ }^{27} \mathrm{P}$ is important, because ${ }^{26} \mathrm{Si}$ destroyed in this way would not end up feeding the isomeric state.

In novae, it has been proposed that the thermal equilibrium between ${ }^{26} \mathrm{Al}$ and ${ }^{26 m} \mathrm{Al}$ can possibly be reached at $\mathrm{T}_{9} \approx 0.4$ [17]. At this range of temperature, the Gamow window lies between 0.2 and $0.5 \mathrm{MeV}$ for the ${ }^{26} \mathrm{Si}(\mathrm{p}, \gamma){ }^{27} \mathrm{P}$ reaction. 


\subsection{The experiment}

For all the reasons explained in section 2.1, we decided to study the ${ }^{26} \mathrm{Si}(\mathrm{p}, \gamma){ }^{27} \mathrm{P}$ using the mirror pair approach: ${ }^{27} \mathrm{P}$ is in fact the mirror nucleus (inverted number of protons and neutrons) of ${ }^{27} \mathrm{Mg}$, and so the proton ANC for the process ${ }^{27} \mathrm{P} \rightarrow{ }^{26} \mathrm{Si}+\mathrm{p}$ can be derived $[14,15]$ as $\left(C_{i j}^{27} P\right)^{2}=R\left(C_{i j}^{27} M g\right)^{2}$, where $R$ can be calculated as $R=\left|\frac{F_{l}\left(i k_{p} R_{N}\right)}{k_{p} R_{n} j_{l}\left(i k_{n} R_{N}\right)}\right|^{2}, F_{l}$ being the regular Coulomb wave function, $j_{l}$ the Bessel function of $l$-th order, and $R_{N}$ the radius of the nuclear interior, while $k_{p}$ and $k_{n}$ are related to the neutron and proton separation energy via the relation $k=\sqrt{\frac{2 \mu \epsilon}{\hbar^{2}}}[14,15]$. Here another relation has been found [14] between $\Gamma_{p}$ and $\left|C_{n}\right|^{2}$ as $\frac{\Gamma_{p}}{\left|C_{n}\right|^{2}}=R_{\Gamma} \approx R_{0}^{r e s}=\frac{\hbar^{2} k_{p}}{\mu}\left|\frac{F_{l}\left(i k_{p} R_{N}\right)}{k_{p} R_{n} j_{l}\left(i k_{n} R_{N}\right)}\right|^{2}$.

We decided then to use the ${ }^{26} \mathrm{Mg}(\mathrm{d}, \mathrm{p})^{27} \mathrm{Mg}$ reaction, using the $19 \mathrm{MeV}$ deuteron beam provided by the U120M cyclotron of the NPI CAS (Řež, Czech Republic), operated by CANAM infrastructure project. About this reaction, a similar attempt using ANC has been made [18] reanalyzing data avaliable in litterature, but the extracted ANC values are in disagreement with other results obtained studying the ${ }^{26} \mathrm{Mg}(\mathrm{t}, \mathrm{d}){ }^{27} \mathrm{Mg}$ reaction [19]. The discrepancies between the two analyses ended up in an S-factor that in the second case is 1.7 times smaller. Further investigations are therefore necessary.

Our experimental set-up was composed by three $\Delta \mathrm{E}$-E telescopes placed at $10^{\circ}$ difference between each other on one side of the beam, and two $\Delta \mathrm{E}-\mathrm{E}$ at $17^{\circ}$ and $37^{\circ}$ on the other side, that were meant as monitors. All the $\Delta \mathrm{E}-\mathrm{E}$ detectors are composed by a $500 \mu \mathrm{m}$ silicon detector for the thin stage and a $5000 \mu \mathrm{m}$ one for the thick one. Angular distributions are measured by moving the detectors to higher angles from the beam axis at fixed steps. The target, provided by the target laboratory of LNS-INFN was composed by $70 \mu \mathrm{g} / \mathrm{cm}^{2}{ }^{26} \mathrm{MgO}$ with a $32 \mu \mathrm{g} / \mathrm{cm}^{2}$ natural carbon backing.

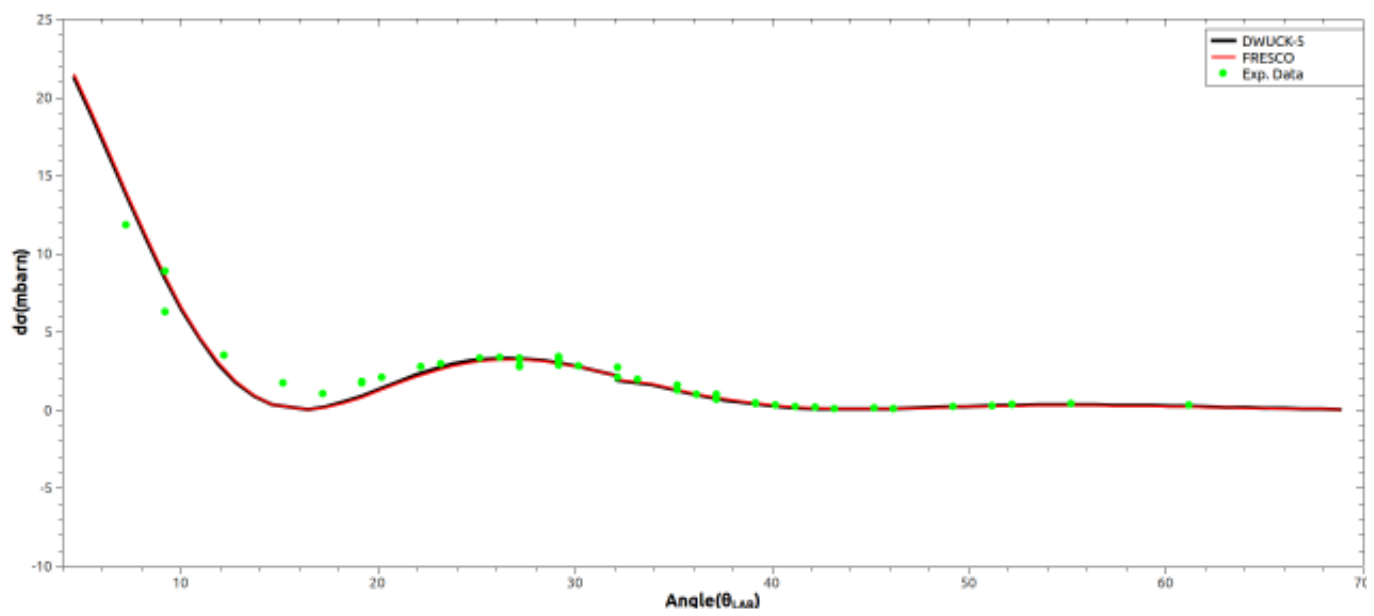

Figure 2. Angular distributions for ground state (same procedure for the first excited state has also been made) for the reaction ${ }^{26} \mathrm{Mg}(\mathrm{d}, \mathrm{p})^{27} \mathrm{Mg}$. The curve is extracted by means of DWBA calculations. More details will be given in a forthcoming paper. 
After particle identification, the resulting optical potential parameters for both elastic scattering ${ }^{26} \mathrm{Mg}+\mathrm{d}$ and ${ }^{26} \mathrm{Mg}(\mathrm{d}, \mathrm{p})^{27} \mathrm{Mg}$ reaction (exit channel) were obtained fitting the angular distribution by means of DWBA calculation (figure 2).

\subsection{Future Work}

With the analysis of the ${ }^{26} \mathrm{Mg}(\mathrm{d}, \mathrm{p}){ }^{27} \mathrm{Mg}$, as for now we were able to obtain information on the behaviour of the angular distribution, on the optical potential parameters necessary for DWBA calculation, and on the SF of the reaction. ANC calculation has yet to be done. Before the extraction of ANC's, peripherality checks must be performed. This part is crucial for the method, given that the reaction must be peripheral. We will apply the standard procedure explained for example in [11]: it consists in a comparison of the modelled $\sigma^{D W B A}$ behaviour for different $\mathrm{R}_{\text {cutoff }}$ of integration. If the process is peripheral, the calculated cross-section must be indipendent on the nucleus interior. Another check that is usually done consists in comparing the radial behaviour of the single-particle wave function $\phi$ with the potential parameter $r_{0}$ [11]. Also, the dependence of $\mathrm{C}^{2}$ on the binding potential involved must be investigated, for example plotting the dependance of it from SPANC $b$. If the variation of $C^{2}$ for increasing values of $b$ is small (usually less than 9\%) than we have another argument for peripherality. Here lies another advantage of the ANC with respect to the SF: the variation of the latter for different values of the SPANC is usually quite severe. Once those checks are performed, the proper ANC calculations will be performed, and mirror pair ANC procedure will be applied to extract the cross-section for the ${ }^{26} \mathrm{Si}(\mathrm{p}, \gamma){ }^{27} \mathrm{P}$ reaction.

\section{Summary}

In this work, the key points of the ANC method are highlighted (section 1), along with an experimental example of an ongoing data analysis (section 2), and some preliminary results have been shown, along with the standard method of extraction of the OMP (through scattering, see section 2.2). Also, the usual ways to check the applicability of the method are discussed (section 2.3).

This work was supported by MEYS Czech Republic under the project EF16_013/0001679

\section{References}

[1] A. M. Mukhamedzhanov and N. K. Timofeyuk, JETP Lett. 51, 282 (1990)

[2] H. M. Xu et al., Phys. Rev. Lett. 73, 2027 (1994)

[3] J. José et al., Astrophys. J. 520, 347 (1999)

[4] O. Koike et al., Astrophys. J. 342, 464 (1999)

[5] V Burjan et al., EPJ A 55, 114 (2019)

[6] A. M. Mukhamedzhanov et. al., Phys. Rev. C. 84, 024616 (2011)

[7] Y. P. Shen et al, Phys. Rev. C. 99, 025805 (2019)

[8] A. M. Mukhamedzhanov \& R. E. Tribble, Phys. Rev. C. 59, 1418 (1999)

[9] A. M. Mukhamedzhanov et. al., Phys. Rev. C. 67, 065804 (2003)

[10] A. M. Mukhamedzhanov et. al., Phys. Rev. C. 78, 015804 (2008)

[11] A.M. Mukhamedzhanov et al., Phys. Rev. C 56, 1302 (1997)

[12] X.D. Liu et al., Phys. Rev. C 69, 064313 (2004)

[13] A. M. Mukhamedzhanov \& A. S. Kadyrov, Phys. Rev. C 82, 051601 (2010) 
[14] N. K. Timofeyuk et al., Phys. Rev. Lett. 91, 232501 (2003)

[15] L. Trache et al., Phys. Rev. C. 67, 062801 (2003)

[16] J. A. Caggiano et al,Phys. Rev. C. 64, 025802 (2001)

[17] A. Coc et al., Phys. Rev. C 61, 015801 (1999)

[18] B. Guo et al., Phys. Rev. C. 73, 048801 (2006)

[19] N. K. Timofeyuk et al., Phys. Rev. C 78, 044323 (2008) 